\title{
A Rapidly Re-Emerging Sexually Transmitted Shigella Flexneri in Man having Sex with the Man (MSM)
}

\author{
Sumit Aggarwal ${ }^{1 * \#}$, Balaji Sivaraman ${ }^{1 \#}$, Deepti Amballkar ${ }^{2}$ \\ ${ }^{1}$ Division of Epidemiology and Communicable Diseases Division, Indian Council of Medical \\ Research, India \\ ${ }^{2}$ Department of Lab Medicine, Max Super Specialty hospital, India
}

*Corresponding author: Dr. Sumit Aggarwal, Scientist C, Epidemiology and Communicable Diseases Division, Indian Council of Medical Research-Headquarters, New Delhi - 110029, India, Tel: 8329944688; Email: aggarwal.sumit@icmr.gov.in

\#Authors equally contributed

\section{Mini Review}

Volume 4 Issue 6

Received Date: October 09, 2020

Published Date: November 24, 2020

DOI: $10.23880 /$ eij-16000171

\section{Abstract}

Sexually transmitted infections (STIs) are widespread across the world and crated a huge health and economic burden to both developing and developed countries. In addition to the established STIs, several bacterial and viral pathogens are emerging with sexually transmittable potential, which attracted global level concern. High-risk sexual network groups such as man having sex with the man (MSM) and human immunodeficiency virus (HIV) are recognized as more vulnerable community in acquiring and transmitting these emerging STIs. Shigella flexneri is one such enteric bacterial pathogen potentially reemerging among MSM community across the world. Although it is easily curable, emergence and transmission of resistant strains among MSM created an additional burden. Thus, the present review discusses a few crucial aspects of re-emerging Shigella flexneri infection in the MSM community.

Keywords: Sexually Transmitted Infection; Man Having Sex with the Man; Enteric Pathogen, Shigella Flexneri; Human Immunodeficiency Virus; Antibiotic Resistance

Abbreviations: STIs: Sexually Transmitted Infections; HIV: Human Immunodeficiency Virus; MSM: Man having Sex with the Man.

\section{Introduction}

About 500 million people are affected by sexually transmitted infections (STIs) annually; thus, it remains a significant global public health challenge [1]. STI makes an enormous burden of morbidity and mortality especially to developing countries, where about $80 \%$ of the cases are reported. Various active and passive surveillance activities and other interventions targeted to hastening case finding, sex education, and effective management are currently practised to mitigate the transmission [2]. However, certain bacterial and viral STIs predominantly exist throughout the globe $[3,4]$. In addition to these established STIs, several infections are silently emerging and re-emerging as STIs and spreading through unprotected sexual activities, especially in the vulnerable groups such as human immunodeficiency virus (HIV) infected people, FSWs and man having sex with the man (MSM) [5]. Thus, it is crucial to identify these pathogens for effective control of STIs. The present review discusses a few crucial aspects of re-emerging Shigella flexneri infection in the MSM community.

\section{Man Having Sex With a Man (MSM) and Associated Sexually Transmitted Infections}

STI is prevalent in the general population as well as high-risk groups like FSW and MSM. MSM has become the more vulnerable community to acquire and spread STIs both 


\section{Epidemiology International Journal}

within and between countries [6,7]. MSM continues as one of the most HIV-susceptible communities at the global level and remains a high-risk group targeted by the National AIDS Control Organization towards mitigating the dissemination of HIV in India [8]. In addition to HIV, several other STIs outbreaks have continuously occurred in this high-risk group that includes chlamydia, gonorrhoea and syphilis [9]. Apart from these STIs, enteric pathogens such as hepatitis A, Shigella, and Giardia commonly affect the MSM community [10].

\section{Is Shigella Sexually Transmittable?}

Among enteric pathogens, Shigella is a highly infectious organism where the ingestion of 10 bacterial cells is sufficient to cause the disease [11]. Shigella is a Gram-negative facultative anaerobic bacterium that causes shigellosis, a disease ranging from self-limiting diarrheal illness to severe dysentery [12]. It is estimated that at least $15 \%$ of the global diarrheal cases are due to the Shigella infection, which causes more than one million deaths annually [13]. Among the four clinically important Shigella species, Shigella flexneri is a clinically significant pathogen owing to their higher mortality rate [14]. Besides, among 50 reported serotypes of the genera Shigella, 2a serotype of $S$. flexneri is the most prevalent serotype worldwide [15]. This bacterium primarily transmitted via contaminated food and water that are either direct or indirect contact with infected human faces and commonly affects children who are less than 5 years of age $[10,16]$. However, Shigella was started recognizing as STI after their outbreak among the MSM community in the late $29^{\text {th }}$ century [17]. Moreover, the evolving evidence suggests their sexual transmission among MSM community which necessitated extensive epidemiological studies to contain their dissemination.

\section{The Emergence and Re-Emergence of Shigella flexneri in MSM}

The very first case of sexually transmitted Shigella among MSM and the bisexual group has been reported from San Francisco, USA in 1974 and the proportion of shigellosis among the male population of the city abruptly raised to $70 \%[17,18]$. Within the next 10 years, the overall rate of $S$. flexneri started declining among children, and an increased case ratio among adult males was found [19]. Similarly, although routine Shigella diagnosis lacks information about sexual practices, the widening inequality between the adult male and female case rates strongly suggested male to male sexual transmission [15]. During the end of the 20th century, this bacterium was routinely found in the HIV-positive MSM and resulted in hospitalization and significant complications, such as bacteraemia or acute kidney injury [20].
Since then, the prevalence of this bacterium is rapidly emerging all along with the globe. In 2004 and 2007, respectively, in Germany and the USA, S. flexneri outbreaks were reported among MSM [21,22]. Further, the predominance of $S$. flexneri found in USA and England between 2004 and 2015, indicating the potential reemergence of this bacterium as an STI in the MSM community $[23,24]$. Currently, this bacterium is rapidly emerging among the MSM community, which raised the global level concern to prevent their spread. For instance, the survey between 2012 and 2015 in USA, 2016 and 2019 in UK, 2004 and 2018 in UK, 2015 and 2018 in the UK, 2018 in Poland, 2016 and 2019 in Australia, 2005 and 2011 in Canada, confirmed the potential of $S$. flexneri in affecting MSM community [10,20,25-29].

\section{Epidemiology of Shigella in India}

In India, shigellosis is one of the significant causes of diarrhoea where $S$. flexneri is the most common species among the genus Shigella. Outbreaks have been reported from many part of the country like Kerala, Maharashtra and West Bengal [30]. Though shigellosis seems to be endemic in India, the precise estimations of morbidity and mortality are lacking. Because several reports from the USA and the UK stated that the frequent outbreaks of $S$. flexneri in MSM might increase morbidity significantly [10]. For instance, about $17 \%$ of $S$. flexneri associated morbidity was found among MSM who attended the STIs clinic in the USA, in 2013 [31].

\section{Role of Social and Behavioral Factors in the Spread of Shigella flexneri among MSM}

There are multiple social and behavioral factors associated with the dissemination of S. flexneri in the MSM community. It includes HIV infection, safe-less sex, oralanal contact, chemsex several sex partners, rate of partner exchange and use of online sexual networks and dating apps $[10,12]$. Oral-anal contact and HIV coinfection are considered to the high-risk factor for the sexual transmission of Shigella where $37-75 \%$ of MSM with shigellosis are HIV positive $[21,32]$. In addition to it, risk-taking behaviour such as the use of disinhibiting substances, including alcohol, crystal methamphetamine, and other recreational drugs enhances the Shigella spread among MSM [6].

\section{The Emergence of the Antimicrobial Resistance in Shigella}

Antibiotic treatment is recommended in general for restrained or severe symptoms which in turn reduce the duration and severity of signs and avert health complications associated with shigellosis. However, the inappropriate use of the antibiotics resulted in the development of antimicrobial 


\section{Epidemiology International Journal}

resistance and further disseminated in the MSM community across the globe [15,32]. Notably, the increasing resistance to third-generation cephalosporins and fluoroquinolones, and most recently, azithromycin [33]. Thus, the rapid emergence of antibiotic-resistant $S$. flexneri strains have become a matter of concern and creating hurdles to the control strategies in any limited treatment resources [34]. These resistant strains have been isolated from MSM of several countries that include Canada, USA, England, Australia, England and India [30,34]. Genomic evidence further confirmed the intercontinental spread of resistant strains in MSM [32]. So if antimicrobial treatment is indicated, knowledge of the local antibiogram of circulating Shigella strains is highly required to treat and contain the spread.

\section{Current Challenges and Future Perspective}

The global trend of shigellosis has been running parallel as a diarrhoeal pathogen and potential STI especially in MSM over the years. Thus, it has emerged as one of the important health problems for most countries, owing to its low infectious dose and other escalating risk factors. Albite shigellosis is easily curable as being a self-limiting illness; emerging antimicrobial resistance hampers the treatment of Shigella infection. Interestingly, rapid dissemination of sexually transmitted drug-resistant pathogens among MSM across a wide geographical area has been previously observed for gonorrhoea, HIV, hepatitis C virus, and syphilis. Perhaps, unraveling the common factors that contribute to the public health impact of antimicrobial-resistant STIs in an MSM community across countries would help in developing effective strategies to evade their spread. Overall, both community-level attentiveness and improved surveillance program for STIs along with Shigella may be initiated to identify various existing and upcoming threats and challenges.

\section{Conclusion}

Shigella flexneri is one of the leading cause of diarrhoea associated mortality and morbidity all around the world. Their epidemics among high-risk sexual networks especially in the MSM community are increasing gradually. Thus, it is important to early recognition of this infection in proctitis and diarrhoea patients especially of high risk group. It is also important to understand possible routes of transmissions and their clinical presentation in various groups of population. In addition, the rapidly emerging antimicrobial resistance among Shigella flexneri isolates results in frequent treatment failures. This has raised global level concerns and necessitated the implementation of focused and more intense public health interventions to prevent early identification and control their transmission.

\section{References}

1. Visalli G, Cosenza B, Mazzù F, Bertuccio MP, Spataro $P$, et al. (2019) Knowledge of sexually transmitted infections and risky behaviours: a survey among high school and university students. J Prev Med Hyg 60(2): 84-92.

2. Alhassan RK, Fatawu AA, Yeboah BA, Nyaledzigbor W, Agana S, et al. (2019 Determinants of use of mobile phones for sexually transmitted infections (STIs) education and prevention among adolescents and young adult population in Ghana: implications of public health policy and interventions design. Reproductive Health 16: 120.

3. Pandit N, Chhaya J (2018) Situation analysis of sexually transmitted infections in India. Journal of Integrated Health Sciences 6(1): 3-7.

4. Unemo M, Bradshaw CS, Hocking JS, de Vries HJ, Francis SC, et al. (2017) Sexually transmitted infections: challenges ahead. The Lancet Infect Dis 17(8): 235-279.

5. Williamson DA, Chen MY (2020) Emerging and Reemerging Sexually Transmitted Infections. New England Journal of Medicine 382(21): 2023-2032.

6. Mayer KH (2011) Sexually transmitted diseases in men who have sex with men. Clin Infect Dis 53(3): 79-83.

7. Gama A, Martins MO, Dias S (2017) HIV research with men who have sex with men (MSM): advantages and challenges of different methods for most appropriately targeting a key population. AIMS Public Health 4(3): 221-239.

8. Garg T, Chander R, Jain A, Barara M (2012) Sexually transmitted diseases among men who have sex with men: A retrospective analysis from Suraksha clinic in a tertiary care hospital. Indian J Sex Transm Dis AIDS 33(1): 16-19.

9. Werner RN, Gaskins M, Nast A, Dressler C (2018) Incidence of sexually transmitted infections in men who have sex with men and who are at substantial risk of HIV infection-A meta-analysis of data from trials and observational studies of HIV pre-exposure prophylaxis. PLoS One 13(12): 0208107.

10. Richardson D, Devlin J, Fitzpatrick C, Pinto Sander N (2020) Sexually transmitted Shigella flexneri and Shigella sonnei in men who have sex with men. Sexually Transmitted Infections.

11. Keay R, Singh G, Abdul Latif M, Rayment M, Nelson M (2014) Shigella flexneri enteritis in risk-taking HIV- 
infected MSM. J Infect 68(1): 103-104.

12. Nisa I, Qasim M, Yasin N, Ullah R, Ali A (2020) Shigella flexneri: an emerging pathogen. Folia Microbiologica 65(2): 275-291.

13. Aslam A, Okafor CN (2019) Shigella (Shigellosis). In StatPearls [Internet], Stat Pearls Publishing.

14. Gentle A, Ashton PM, Dallman TJ, Jenkins C (2016) Evaluation of molecular methods for serotyping Shigella flexneri. J Clin Microbiol 54(6): 1456-1461.

15. Baker KS, Dallman TJ, Ashton PM, Day M, Hughes G, et al. (2015) Intercontinental dissemination of azithromycinresistant shigellosis through sexual transmission: a cross-sectional study. Lancet Infect Dis 15(8): 913-921.

16. Gupta A, Polyak CS, Bishop RD, Sobel J, Mintz ED (2004) Laboratory-confirmed shigellosis in the United States, 1989-2002: epidemiologic trends and patterns. Clin Infect Dis 38(10): 1372-1377.

17. Dritz SK, Back AF (1974) Shigella enteritis venereally transmitted. N Engl J Med 291(22): 1194.

18. Bader M, Pedersen AH, Williams R, Spearman J, Anderson $\mathrm{H}$ (1977) Venereal transmission of shigellosis in SeattleKing county. Sex Transm Dis 4(3): 89-91.

19. Tauxe RV, McDonald RC, Hargrett Bean N, Blake PA (1988) The persistence of Shigella flexneri in the United States: the increasing role of adult males. Am J Public Health 78(11): 1432-1435.

20. Allen H, Mitchell HD, Simms I, Baker KS, Foster K, et al. (2020) Evidence for reinfection and persistent carriage of Shigella species in adult males reporting domesticallyacquired infection in England. Clin Microbiol Infect.

21. Marcus U, Zucs P, Bremer V, Hamouda O, Prager R, et al. (2004) Shigellosis-a re-emerging sexually transmitted infection: an outbreak in men having sex with men in Berlin. Int J STD AIDS 15(8): 533-537.

22. Surawicz CM (2007) Shigella: a sexually transmitted infection in men who have sex with men. Gastroenterology 133(5): 1737-1738.

23. Simms I, Field N, Jenkins C, Childs T, Gilbart VL, et al. (2015) Intensified shigellosis epidemic associated with sexual transmission in men who have sex with menShigella flexneri and S. sonnei in England, 2004 to end of February 2015. Euro Surveill 20(15): 21097.

24. Foodborne Diseases Active Surveillance Network (FoodNet). CDC.

\section{Epidemiology International Journal}

25. Wilmer A, Romney MG, Gustafson R, Sandhu J, Chu T, et al. (2015) Shigella flexneri serotype 1 infections in men who have sex with men in Vancouver, Canada. HIV Med 16(3): 168-175.

26. Guglielmino CJ, Kakkanat A, Forde BM, Rubenach S, Merone L, et al. (2020) Outbreak of multidrug-resistant (MDR) Shigella flexneri in northern Australia due to an endemic regional clone acquiring an IncFII plasmid. European J Clin Microbiol Infect Dis.

27. Bardsley M, Jenkins C, Mitchell HD, Mikhail AF, Baker KS, et al. (2020) Persistent Transmission of Shigellosis in England Is Associated with a Recently Emerged Multidrug-Resistant Strain of Shigella sonnei. J Clin Microbiol 58(4).

28. Stażyk K, Krycińska R, Jacek C, Garlicki A, Biesiada G (2019) Diarrhoea caused by Shigella flexneri in patients with primary HIV infection. Int J STD AIDS 30(8): 814816.

29. Eikmeier D, Talley P, Bowen A, Leano F, Dobbins G, et al. (2020) Decreased Susceptibility to Azithromycin in Clinical Shigella Isolates Associated with HIV and Sexually Transmitted Bacterial Diseases, Minnesota, USA, 2012-2015. Emerg Infect Dis 26(4): 667-674.

30. Taneja N, Mewara A (2016) Shigellosis: epidemiology in India. Indian J Med Res 143(5): 565.

31. Cresswell FV, Ross S, Booth T, Pinto Sander N, Alexander E, et al. (2015) Shigella flexneri: a cause of significant morbidity and associated with sexually transmitted infections in men who have sex with men. Sex Transm Dis 42(6): 344 .

32. Baker KS, Dallman TJ, Field N, Childs T, Mitchell H, et al. (2018) Genomic epidemiology of Shigella in the United Kingdom shows the transmission of pathogen sublineages and determinants of antimicrobial resistance. Sci Rep 8(1): 1-8.

33. Chiou CS, Izumiya H, Kawamura M, Liao YS, Su YS, et al. (2016) The worldwide spread of ciprofloxacin-resistant Shigella sonnei among HIV-infected men who have sex with men, Taiwan. Clin Microbiol Infect 22(4): 383.11383.16.

34. Ranjbar R, Farahani A (2019) Shigella: antibioticresistance mechanisms and new horizons for treatment. Infect Drug Resist 12: 3137-3167. 